\title{
CAFCLA: A Conceptual Framework to Develop Collaborative Context-Aware Learning Activities
}

\author{
Óscar García, Ricardo S. Alonso, Dante I. Tapia, Elena García, \\ Fernando De la Prieta, and Ana de Luis \\ Department of Computer Science and Automation, University of Salamanca. Plaza de la \\ Merced, s/n, 37008, Salamanca, Spain \\ \{oscgar, ralorin, dantetapia, elegar, fer, adeluis\} @usal.es
}

\begin{abstract}
Advances appeared in Information and Communication Technologies along last years have given raise to new interaction ways between people and technology. Ambient Intelligences (AmI) is a multidisciplinary research area which promotes the use or technology in a transparent way to facilitate everyday tasks. Education is one field that benefits from AmI: collaboration between students in innovate ways, data acquisition from the context or real time location systems enhance learning processes. This paper presents CAFCLA, a framework aimed at designing, developing and deploying AmI-based educational scenarios where collaboration between students and contextual information are available every time and everywhere through multiple resources and communication protocols.
\end{abstract}

Keywords: Ambient Intelligence, Computer Supported Collaborative Learning, Context-aware Learning, Wireless Technologies, Real Time Locating Systems.

\section{Introduction}

In recent years there has been a technological explosion that has flooded our society with multiple and different technological devices. Similarly, devices improve their processing and storage capacity and storage, their user interfaces or their communication skills day by day. Thanks to these advances, we are currently surrounded by technology that has changed our habits and customs [1]. All this has given cause to appear fields such as Ambient Intelligence, whose main objective is to simplify the use of technology to improve the quality of life of users [2].

Education is one of the areas in which Ambient Intelligence presents a greater potential as it provides new ways of interaction and communication between individuals and technology systems [3]. The usage of Communication and Information Technologies (ICT) has been present in educational innovations over recent years [4], modernizing the traditional transmission of content through electronic 
presentations, email or more complex learning platforms such as Moodle ${ }^{1}$ or $\mathrm{LAMS}^{2}$ and fostering collaboration between students (Collaborative Learning) [5]. Besides the use of those general-purpose tools in education, other tools that make more concrete use of technology have appeared. This applies to those that make use of Context-awareness information and ubiquitous computing and communication, fundamental parts of Ambient Intelligence [6].

The inclusion of Context-awareness in educational environments and processes refers to Context-aware Learning [7], a particular area of application of Contextaware Computing [8]. Moreover, being able to know, characterize and customize the context that surrounds a leaning situation at a time and place allows flexibility in the education process, so learning does not only occurs in classrooms, but in a museum, park or any other place [9], obtaining ubiquitous learning spaces. Thus, there is an extensive literature that addresses the problem of this kind of learning, highlighting those works that attempt to solve contextual information acquisition and providing data to users $[10,11,12]$. The use and integration of different technologies and the approach to specific learning activities characterize analyzed solutions. However, there is a lack of solutions that attempt to facilitate the work of educators. The complexity of understanding and use of the technology and solutions in the aforementioned works does not allow a wide use of them.

This paper presents a conceptual framework aimed at designing, developing and deploying AmI-based educational scenarios. Educators are able to characterize the context where the learning activity will occur through the creation of a world model in which locate data collectors (e.g. sensors), indentify and characterize areas of interest (e.g. paintings in a museum), etc. Moreover, the collaboration between students and the customization of the information available is also provided and can be integrated in the activity design.

The following section describes the background and problem description related to the approach presented. Then, the main characteristics of the framework proposed are described: what kind of activities is covered, how educators can create an activity, what technologies are used, which parts compose the framework and how they work together. Finally, the conclusions and future work are depicted.

\section{Background and Problem Description}

A growing interest in educational software, commonly known as e-learning, has appeared over recent years [13]. Among the wide range of existing educational software are CSCL (Computer Supported Collaborative Learning) applications [14]. A collaborative learning system consists of a set of tools that facilitate the implementation, development and deployment of learning activities. Those activities allow different ways of interaction between participants involved that activate learning mechanisms [15]. CSCL has become an important research field within

\footnotetext{
${ }^{1}$ http://moodle.org

${ }^{2} \mathrm{http} / / /$ lamsfoundation.org/
} 
education that attracts different interests from the purely educational to those focused on improving human-computer interaction [5].

On the other hand, contextual information includes any data that can be used to characterize a person, place or object that is considered relevant to the interaction between users, user and applications or systems or between systems or applications [16]. In addition to the relevant information that context provides, it is important to consider other parameters that relevantly affect this type of information, such as identification, time and location [6]. The information exchange taking place between technology and users, in order to contextualize an environment in which learning takes place, and customize the content of the learning activity can be understood as collaboration. Thus, Context-aware Learning must take into account the interactions between people and the different technological components of the system in all its combinations.

\subsection{Providing Context-Aware to Learning}

Providing contextual information and fostering collaboration between students benefit the learning process [3]. Moreover the combination of Collaborative and Context-aware Learning naturally leads to think about ubiquitous learning spaces, characterized by "providing intuitive ways for identifying right collaborators, right contents and right services in the right place at the right time based on learners surrounding context such as where and when the learners are (time and space), what the learning resources and services available for the learners, and who are the learning collaborators that match the learners' needs" [17].

A better understanding of environment through technology allows educators to customize the content provided to students. Similarly, technology facilitates the interaction with the environment and between students. This should try to be reached in a way as transparent and ubiquitous as possible. The technologies used for the collection of contextual information and for the communication between different devices are the cornerstone of the different works presented here. Literature about Context-aware Learning proposals has been deeply reviewed in this work. Some of the most representative works are classified in this paper, following technological criteria related to communications and data collection.

A first approach to provide contextual information is "tagging the context". RFID (Radio Frequency IDentification) is the most spread technology [18]. While other technologies such as NFC (Near Field Communication) [19] or QR Codes (Quick Response Codes) [20] are growing fast. As can be seen in the usage of Active RFID, location and context-awareness are closely related: knowing precisely location of objects and people allow determine what is surrounding them and, consequently, characterize the context they are involved. GPS (Global Positioning System) is the most used technology to provide location in Context-aware Learning [20, 21, 22]. This location system provides a high accuracy level and is currently implemented in a wide range of smart phones and mobile devices. In those cases, the mobile device provides a position to the system. Those solutions are used in different scenarios as planning routes [22] or manage a student scheduler [21]. However, most of those works do not implement a concrete use case, but 
propose a general purpose model in which GPS technology is included to facilitate the provision of contextual data.

Moreover, GPS technology does not work indoors because of the direct vision necessary between satellites and devices. Indoor environments are very common in learning: museums, laboratories or the school are places where activities that requires mobility can be developed. Trying to cover this lack location systems that user Active RFID [18] or Wi-Fi [12] are used. Both cases the performance of systems is similar: student's position y determined by the access point is giving coverage in this moment. This type of approach has significant limitations when developing context-aware learning activities: location accuracy is poor. This situation presents an important problem when areas where context information is different are close (e.g. two paintings in a museum).

Changing the way of contextualize the environment where the learning activity occurs, some tendencies use sensor networks [12]. A sensor is an electrical or mechanic device that measures a physical magnitude. In this case environmental characterizations is reduced to those data provided by the sensors. In order to make the learning process more transparent to students, ad-hoc networks are considered to collect and transport data from sensors to remote points [11]. This networks facilitate the connection between devices anytime and anywhere without a previous infrastructure.

\subsection{Problem Description}

The review of the literature evidences some lacks in the Context-aware Learning systems proposed until now. Even some works try to combine different technologies to cover as much situations as possible [12], most of them only cover specific learning situations, as those where tagging context with RFID/NFC [19] is necessary or those where learning occurs outdoors [22]. The combination of both situations is only addressed by M2learn [12]. However, this solution does not provide a precise and efficient Real Time Locating System or the possibility to integrate wireless sensor networks, excepts RFID systems.

On the other hand, none of the solutions mentioned before takes into account Ambient Intelligence issues. The proposed solutions focus their work on the architectural description, framework integrators or end-user applications whose designs have not taken into account how complex will be them for educators or students. Some aspects such as designing intuitive and attractive interfaces or abstracting end users from the complexity of technology, issues on which Ambient Intelligence pays special attention, are not taken into account. Thus, if these aspects are excluded from the solution design process, the final result may be rejected by students and educators.

For this reason, the design process must take into account, from the beginning, the opinion of all the stakeholders [5], educators, designers and developers. This way is easier to accomplish with Ambient Intelligent issues related to user interfaces and usability of final applications.

Moreover, works analyzed in this review do not include mechanisms for data or communication management. Ambient Intelligence emphasizes the transparency 
of technologies for users. In addition, technology is used to ease ordinary task or improve activities and quality of life. In this sense, systems that combine different technologies do not facilitate mechanisms to change between them (e.g. different communication protocols) attending to the needs of a situation. Similarly, data have to be managed in an intelligent and efficient way. Most of the literature reviewed does not include this issue, using only standard data repositories that only consider persistency and consistency [29]. Functionalities like data redundancy to solve network failures helps make the system dynamic and benefits data accessibility with independence of the place and the moment.

Collaboration between students is another issue not considered by many proposals [11]. It is well known that collaboration benefits the learning process [3]. Including mobile devices and wireless communication protocols in any learning design that requires mobility (as discussed in this paper) is nowadays necessary. Mobile devices easily connect each other so including collaboration between students is easy, increasing the variety of activities and improving learning process.

Furthermore, this work is developed following Ambient Intelligence issues. Some of those issues, as personalization of context provided or transparency and ease of use for educators and users are vaguely taken into account. Moreover, the inclusion of reasoning mechanisms facilitate the personalization of data provision or the communication management of this kind of complex systems [23]. The present work considers all these issues when describing and designing the framework presented next.

\title{
3 CAFCLA: Context-Aware Framework for Collaborative Learning Applications
}

\begin{abstract}
CAFCLA (Context-Aware Framework for Collaborative Learning Applications) is a framework aimed at designing, developing and deploying AmI-based educational scenarios, focusing in collaborative and context-aware activities. The framework integrates a set of wireless context-aware technologies (e.g. GPS, ZigBee, Wi-Fi, or GPRS/UMTS). Those technologies allow establishing collaborative activities based on Ambient Intelligence among students and teachers. In this sense, communication models vary dynamically depending on the activity; for example, following a client-server model to perform a data query or forming an adhoc network to gather contextual information. Thus, the contextual information is always available and may be modified every time.
\end{abstract}

\subsection{Main Characteristics}

As mentioned before, the biggest challenge of CAFCLA is to ease the design, develop and deployment of Ambient Intelligences educational applications in which contextual data and collaboration are required. This implies that educators are able to choose what, where and how they want to develop a collaborative learning activity using contextual data. The main resources that CAFCLA offers to educators in order to reach these objectives are: 
- Identification: each participant or object that takes part in the learning activity is uniquely identified. This way the learning process can be customize for each student: once the environment is characterize, educator can choose what information is given to each student, filtering data in order to the student's identification. Moreover, educators can create profiles for groups of students in order to create group activities or manage information in an easy way.

- Wireless Sensors Network: educators can collect environmental data through sensors. CAFCLA integrates n-Core ${ }^{\circledR}$ ZigBee wireless sensor network [24]. n-Core ${ }^{\circledR}$ platforms consists of a set of wireless devices with reduced energy consumption and physical size. Each of them collects one or more physical magnitude used to characterize the environment. They use ZigBee communication.

- GPS Localization: to contextualize environments a provide users' position outdoors, CAFCLA integrates GPS localization.

- ZigBee Real Time Location System: to solve the inability to provide indoor location of GPS, CAFCLA integrates n-Core ${ }^{\circledR}$ ZigBee Real Time Location System [24]. The deployment of this systems consists of a set of beacons strategically located along the area that needs localization. Its accuracy, under 1 meter, allows educators to define as many areas of interest as they desire in which customize contents of includes environmental information.

- Collaboration: thanks to the different communication protocols implements by the used devices. students are able to collaborate between them through different activities proposed by educators. Moreover, the uniquely identification of each student can be used by educator to decide who collaborate with whom, so they can control at any moment the develop of the activity.

- Disconnected operation mode: a failure in the connection to the Internet can avoid data or activity access and, consequently, stop the activity. As ZigBee protocol is integrated in CAFCLA, devices are able to form ad-hoc networks to communicate each other (as sensors do) without the requirement of a previous infrastructure. Students can collaborate each other using this kind of networks.

- Analysis interaction: ad-hoc networks, outdoors and indoors location and uniquely identification of each student provides interesting information about interactions between students that can be analyzed in order to evaluate and improve the learning process.

This set of resources allows that educators only think in the learning process, abstracting the technological layer and the complications and difficulties that this introduces. CAFCLA provides educators an application in which graphically design the educational scenario where the activity is taking place. Once collected the basic data, the framework tells the educator how many and what kind of devices are needed, where each of them should be located, what services are necessary to use and how and where insert contextual data into the system. On the other hand, CAFCLA provides developers with programming functions that they have to use to develop the application designed. Thus, developers can abstract from any problems of logical, devices, configurations, etc. 


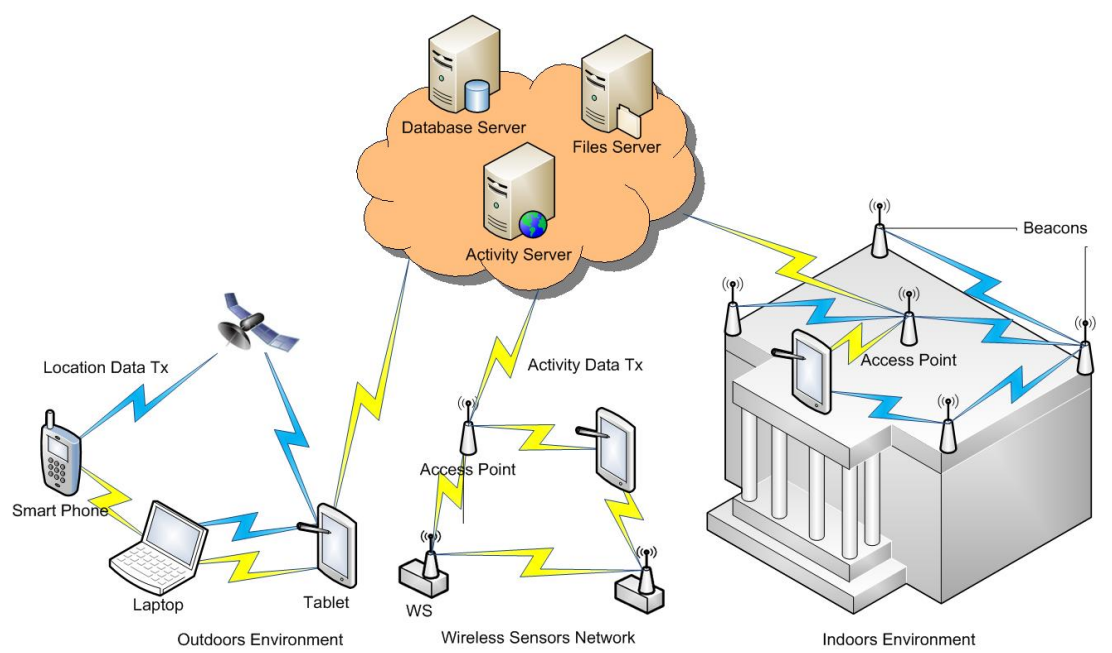

Fig. 1 Basic architecture of CAFCLA.

\subsection{Architecture}

As can be seen in Figure 1, three are basic cases for using CAFCLA: learning outdoors, learning indoors and learning using wireless sensor networks. All of them can be used at the same time to support the same application. Moreover, data management is located in one or more servers. The physical components of each case and it basic performance are as follows:

- Outdoors environment: this functionality requires, at least, a GPS provided device. Moreover a maps platform (e.g. Google Maps) is needed. When designing activities, educators draw a concrete area in the map. Then includes al the contextual information related to that area, including different versions of the information to be used in different activities. Then the system associate an area to one or more descriptions. Then, when students are developing the activity GPS embedded in the device is continuously transmitting its position. When the student enters into an area he receives contextual information accordingly to the design of the activity.

- Indoors environment: $n$-Core ${ }^{\circledR}$ platform ease the location process. The zone where location system is implemented is provided with a set of beacons ( $\mathrm{Si}$ rius $\mathrm{D}$ ). Those beacons are able to communicate each other and send information about the location of a student to the access point, Each student is provided with a ZigBee device (Sirius B) that communicate with its closer beacons that gather different data and send it to the access point. The access point send all the data information to the activity server where a location engine calculate the position of the student. The way contextual information is included in the system is identical to the previous case. 
- Wireless Sensors Network: $n-C o r e \circledR$ also provides different wireless sensors (e.g. temperature or pollution) that form a network infrastructure through which data is sent to the access point that send the information to the data server. Moreover, sensors can connect with other ZigBee devices (e.g. a laptop provided with a Sirius B device) and share it data with them. In this particular case, educators must decide the location of each sensor and the type of data and frequency they gather it.

- Activity management: this block manages all the activities and provides data and content to users and activities. It is composed of one or more servers that provides any information request by students or activities. This modules is deeper described in section 3.4.

\subsection{Communication}

CAFCLA implements three different communication protocols that serve as basis for all the communications of the framework:

- Wi-Fi: most of mobile devices, as laptops, tablets or smart phones are provided with Wi-Fi communication. Moreover, Wi-Fi internet access is provided in great part of the placer where daily life takes place, such as homes, schools, universities, coffee shops, museums even public spaces as parks or places. thus, it is easy to access to remote resources at any time.

- GPRS / UMTS: CAFCLA provides the possibility to use 3G communications to access the resources of the learning activity. For these places where a Wi-Fi Internet connections is not available, if the student's device has 3G connection, he is able to access to any resource.

- ZigBee: besides the connections provided by Wi-Fi and GPRS capabilities to access to remote resources, CAFCLA also includes the possibility to integrate ZigBee communication protocol. This is helpful when deploying ad-hoc networks that foster peer-to-peer collaboration between students without the needed of having Internet access or when a device gather data from different sensors.

CAFCLA integrates these protocols so that the communication between devices and people is transparent both to the design activity (taking as constraints only the functionalities of the framework and not technological ones) as to when carrying out specific activities.

In addition to integration of these communication protocols in the framework, the biggest challenge of CAFCLA is to manage them in an intelligence and effective way. Thus, the network management system hides the complexity of decide which are the available networks, select the most appropriate network depending of the activity and the availability and other tasks that must be transparent to educators and students, such as changing the communication protocol when necessary. He is responsible to detect at any time which communication networks are available, choose the most appropriate to develop the activity, offer and provide peer communication (e.g. sensor-device or two students to collaborate). 


\subsection{Data Management}

Once defined all the communications protocols involved in the framework it is necessary to introduce how information and activities are managed. In this sense, management has been divided in three main parts:

- Activity Server: this server is responsible for maintaining a general repository with all the activities and services developed using CAFCLA. Thus, all of them are reusable by other people.

- Database Server: provides all the logical information of the system and subsystems developed. It has a structure of great directory that stores and receive queries such as services used in activities, customized information, contextualization of environments, location or sensor networks available, etc.

- Files Server: data is completely separated from logical structure. A file repository have been designed in order to maintain integrity, security, redundancy and availability of the data.

Access to this information is marked by the pattern followed to develop the activity designed. A simple reception of contextual information accesses the different servers as follows: one access point transmits the data necessary to calculate the position of a student to the Activity Server, more specifically the service capable of calculate its position. Once the position is calculated and passed to the manager of the activity (responsible for maintaining a logical flow of it), it requests the data context for this position, querying the Database Server. This server will refer to specific data that may be obtained from the File Server.

On the other hand, systems designed using CAFCLA must be dynamic and must work sometimes without an Internet access. For this reason a data management system is designed and developed. It is responsible to decide which data should be stored in each device depending of the location where the activity is taking place and the resources there available.

\section{Conclusions and Future Work}

Communication and Information Technologies are in constant rise from several years ago. Different devices and forms of communication have appeared and have been adopted in daily life naturally. However, much work is necessary to manage and coordinate all the elements involved until raise an end-user solution.

Education is a field where technology use is widespread. The use of new mobile devices, context-aware technologies or communication protocols promote the emergence of new learning scenarios. More specifically, location technologies (e.g. GPS) or wireless communication protocols (e.g. Wi-Fi or GPRS) are included in a large number of proposed solutions. However, they are usually focused to cover concrete scenarios, so it is difficult to extrapolate solutions from a scenario to another.

Moreover, the design of aforementioned solutions are usually made from a highly technical point of view. A global understanding of the learning process 
requires the participation of all the stakeholders, educators, designers and developers. Collaboration among them is an essential part for the success of the proposal. Furthermore, all stakeholders participation in the whole process benefits the inclusion of the solution in a wider range of learning scenarios.

Then, it is necessary to design and develop a set of tools that provides a basis to easily design, develop and deploy the learning activities this works refers. This paper presents CAFCLA as an example of this kind of tools. CAFCLA is a framework that integrates different context-aware technologies, real time location systems and communication protocols to abstract educators and developers of learning activities on the complexity of the simultaneous use of different technologies involved. In this case, CAFCALA is focused on facilitating the design and develop of collaborative context-aware learning activities.Future work includes the inclusion of management systems in the framework. Two will be necessary: first, a communication management system that will provides each moment the most appropriate communication protocol to be used. In second place, a data management system will be also integrated to improve the availability of resources and anticipate to situations in which general purpose resources are not accessible. By the moment, Multi-agent Systems is the technology that has considered more suitable. However, a deep analysis and comparison will be done in order to objectively determinate the technology that will be used.

Acknowledgments. This project has been supported by the Spanish Ministry of Science and Innovation (Subprograma Torres Quevedo).

\section{References}

1. Jorrín-Abellán, I.M., Stake, R.E.: Does Ubiquitous Learning Call for Ubiquitous Forms of Formal Evaluation?: An Evaluand oriented Responsive Evaluation Model. Ubiquitous Learning: An International Journal 1 (2009)

2. Corchado, J.M., Bajo, J., de Paz, Y., Tapia, D.I.: Intelligent environment for monitoring Alzheimer patients, agent technology for health care. Decision Support Systems 44(2), 382-396 (2008)

3. García, Ó., Tapia, D.I., Alonso, R.S., Rodríguez, S., Corchado, J.M.: Ambient intelligence and collaborative e-learning: a new definition model. Journal of Ambient Intelligence and Humanized Computing, 1-9 (2011)

4. Scardamalia, M., Bereiter, C., McLean, R.S., Swallow, J., Woodruff, E.: ComputerSupported Intentional Learning Environments. Journal of Educational Computing Research 5(1), 51-68 (1989)

5. Gómez-Sánchez, E., Bote-Lorenzo, M.L., Jorrín-Abellán, I.M., Vega-Gorgojo, G., Asensio-Pérez, J.I., Dimitriadis, Y.A.: Conceptual framework for design, technological support and evaluation of collaborative learning. International Journal of Engineering Education 25(3), 557-568 (2009)

6. Traynor, D., Xie, E., Curran, K.: Context-Awareness in Ambient Intelli-gence. International Journal of Ambient Computing and Intelligence 2(1), 13-23 (2010)

7. Laine, T.H., Joy, M.S.: Survey on Context-Aware Pervasive Learning Environments. International Journal of Interactive Mobile Technologies 3(1), 70-76 (2009)

8. Dey, A.K.: Understanding and Using Context. Personal and Ubiquitous Computing 5(1), 4-7 (2001) 
9. Bruce, B.C.: Ubiquitous learning, ubiquitous computing, and lived experience. In: Cope, W., Kalantzis, M. (eds.) Ubiquitous Learning, pp. 21-30. University of Illinois Press, Champaign (2008)

10. Abowd, G.D., Atkenson, C.G., Hong, J., Long, S., Kooper, R., Pinkerton, M.: Cyberguide: A mobile context-aware tour guide. Wireless Networks 3(5), 421-433 (2006)

11. Chen, T.-S., Yu, G.-J., Chen, H.-J.: A framework of mobile context management for supporting context-aware environments in mobile ad hoc networks. In: Proceedings of the 2007 International Conference on Wireless Communications and Mobile Computing, pp. 647-652 (2007)

12. Martín, S., Peire, J., Castro, M.: M2Learn: Towards a homogeneous vision of advanced mobile learning development. In: 2010 IEEE Education Engineering (EDUCON), pp. 569-574 (2010)

13. Clark, R.C., Mayer, R.E.: E-Learning and the Science of Instruction: Proven Guidelines for Consumers and Designers of Multimedia Learning. John Wiley \& Sons (2011)

14. Dillenbourg, P.: What do you mean by Collaborative Learning? Collaborative Learning. In: Cognitive and Computational Approaches, pp. 1-19. Elsevier Science Ltd, Oxford (1999)

15. Koschmann, T.: CSCL: Theory and practice of an emerging paradigm. Lawrence Erlbaum, Mahwah (1996)

16. Abowd, G.D., Dey, A.K., Brown, P.J., Davies, N., Smith, M., Steggles, P.: Towards a Better Understanding of Context and Context-Awareness. In: Gellersen, H.-W. (ed.) HUC 1999. LNCS, vol. 1707, p. 304. Springer, Heidelberg (1999)

17. Yang, S.J.H.: Context Aware Ubiquitous Learning Environments for Peer-to-Peer Collaborative Learning. Educational Technology \& Society 9(1), 188-201 (2006)

18. Hwang, G.-J., Yang, T.-C., Tsai, C.-C., Yang, S.J.H.: A context-aware ubiquitous learning environment for conducting complex science experiments. Computers \& Education 53(2), 402-413 (2009)

19. Blöckner, M., Danti, S., Forrai, J., Broll, G., De Luca, A.: Please touch the exhibits!: using NFC-based interaction for exploring a museum. In: Proceedings of the 11th International Conference on Human-Computer Interaction with Mobile Devices and Services, MobileHCI 2009, vol. 71, pp. 1-2 (2009)

20. Tan, Q., Kinshuk, Kuo, Y.-H., Jeng, Y.-L., Wu, P.-H., Huang, Y.-M., Liu, T.-C., et al.: Location-Based Adaptive Mobile Learning Research Framework and Topics. In: International Conference on Computational Science and Engineering, CSE 2009, vol. 1, pp. 140-147 (2009)

21. Saccol, A.Z., Kich, M., Schlemmer, E., Reinhard, N., Barbosa, J.L., Hahn, R.: A Framework for the Design of Ubiquitous Learning Applications. In: 42nd Hawaii International Conference on System Sciences, HICSS 2009, pp. 1-10 (2009)

22. Driver, C., Clarke, S.: An application framework for mobile, context-aware trails. Pervasive and Mobile Computing 4(5), 719-736 (2008)

23. Padovitz, A., Loke, S., Zaslavsky, A.: The ECORA framework: A hybrid architecture for context-oriented pervasive computing. Pervasive and Mobile Computing 4(2), 182215 (2008)

24. Nebusens, n-Core ${ }^{\circledR}$ : A Faster and Easier Way to Create Wireless Sensor Networks. n-Core ${ }^{\circledR}$ (2012), http: / / www.n-core. info (last access: January 18, 2012) 